\title{
Neosilbersalvarsan (NSS.) und seine einzeitige Verwendung mit Novasurol.
}

Von Dr. Georg Krebs, Spezialarzt für Hautkrankheiten in Leipzig.

Ende Mai 1920 sandte mir Geh.-Rat Kolle 500 Röhrchen Neosilbersalvarsan in Stärken von 0,2 bis 0,5 mit der Bitte, dieses neue Salvarsanpräparat auf seine Wirksamkeit, seine Verträglichkeit und seine Heilerfolge hin' zu studieren. Den gesammeiten Erfahrungen liegt ein Material von 200 Patienten ailer Stadien der Syphilis und eine Zeit von 13 Monaten zugrunde. Behandelt wurden:

a) 95 Fälle im primären, nach Wassermann (und meist auch Sachs-Georgi) noch negativen Stadium, bei denen die Infektion höchstens 3 Wochen zurǘcklag.

b) 52 Fälle mit bereits positivem Wassermann $(t=t+)$, bei denen außer dem Primäraffekt noch keinerle1 Haut- oder Schleimhauterscheinungen nachweisbar waren.

c) 36 Fälle im vorgeschrittenen Sekundärstadium mit +++ $=+++$ Wassermann und ausgesprochenen Haut- bzw. Schleimhauterscheinungen.

d) 17. 1 älle von Spätsyphilis mit negativem und positivem Wassermann.

Die Behandlung wurde - abgesehen von einigen wenigen a us schli e Blich mit NSS. behandelten Fällen - in einzeitiger Kombination von NSS. mit Novasurol durchgeführt, und zwar nach folgendem Durchschnittsschema, von dem selbstredend je nach Lage der Dinge hin und wieder abgewichen wurde und abgewichen werden muBte. (Stomatitis, die übrigens merkwürdigerweise trotz reichlichster $\mathrm{Hg}$ Zufuhr nur bei einem verschwindend kleinen Prozentsatz der Patienten auftrat, stärkere Reaktionserscheinungen, Dermatitiden usw.)

1. Injektion NSS. 0,2 , Toleranzprïfung

2. Injektion NSS. 0,2 , Novasurol 1,0

3. Injektion NSS. 0,3, Novasurol 1,0

4. Injektion NSS. 0,3 , Novasurol 1,5

5. Injektion NSS. 0,3 , Novasurol 1,5

Es sind demnach im allgemeinen für eine Kur $4,8 \mathrm{~g}$ NSS und $24 \mathrm{ccm}$ Novasurol verwendet worden. Die Injektionen sind im Verfolg des vorstehenden Schemas in der ersten Zeit der 13 monatlichen Behandlungsära mit Zwischenräumen von 3 , später von 2 Tagen, in den letzten Monaten sogar einen Tag um den anderen ausgeführt worden. Abgesehen von einigen belanglosen Störungen im Allgemeinbefinden einiger weniger Patienten, sind diese sehr kurzen Intervalle zwischen den einzelnen Einspritzungen durchweg gut vertragen worden. Und ich bin der Ansicht, dal $3^{3}$ besonders bei den abortiv zu behandelıden, aber auch bei den sekundären Fällen der Syphilis diese Kumulativwirkung von hervorragender Bedeutung für die restlose Erfassung der Spirochäten ist. Jedenfalls spricht die Möglichkeit der Einverleibung derartiger Dosen in so kurzen Zwischenräumen für die geringe Toxizität des neuen Präparates.

Die Verträglichkeit war ausgezeichnet, auffallenderweise aber nur bis zu der Dosis 0,4 . Bei 25 mit 0,5 behandelten Kranken beiderlei Geschlechts haben wir in den meisten Fäilen entweder in unangenehmer Weise auftretenden angioneurotischen Symptomenkomplex, Brechreiz und Erbrechen kurz nach der Injektion oder momentan sich einstellende heftige Kopfschmerzen beobachtet. Wir befinden uns bezüglich dieser Beobachtung in Uebereinstimmung mit den Erfahrungen, die Hannes Weber und $\mathrm{Erna} H \mathrm{H}$ ibenberg in der Klinik von Prof. Klie neberger in-Zittau (Derm. Wschr. 1921 $\mathrm{Nr} .35$ ) gemacht haben. Auch sie haben bald die Verwendung von 0,5 NSS. unterlassen. Ganz leichte Formen von angioneurotischem Symptomenkomplex haben wir auch bei 0,3 und 0,4 NSS. beobachtet, doch waren diese Erscheinungen so vorübergehend, daß sie als belanglos bezeichnet werden können. Ebenso harmlos waren die hin und wieder - besonders an den Armen und Händen - auftretenden Dermatitiden, die bei Aussetzung der Behandlung schnell verschwanden, ohne später wiederzukehren, sodaß die Kur zu Ende geführt werden konnte. Bemerkenswert sind die sehr urangenehmen Zufälle, die wir bei zwei weiblichen Patienten mittleren Alters gleich nach der ersten Injektion zu beobachten. Gelegenheit hatten; starker angioneurotischer Symptomenkomplex, Schwindelgefühl, Brechreiz und tagelang anhaltende Schwäche, in dem einen Fall verbunden mit universeller Dermatitis und tagelang anhaltendem Fieber. Es hat sich đann aber später herausgestellt, daß beide eine ausgesprochene Arsenidiosynkrasie hatten, selbst auf die kleinsten Dosen anderer Salvarsanpräparate $(0,1 \mathrm{Neo}$ - und 0,15 Silbersalvarsan) reagierten sie mit ähnlichen Symptomen. Bei beiden wurde dann die Kur mit intravenösen Novasurolinjektionen $\mathrm{zu}$ Ende geführt, die anstandslos vertragen wurden.

Viele Patienten, besonders solche im bereits vorgeschrittenen Sekundärstadium, die körperlich recht reduziert waren, rühmten eine auffällig schnelle Besserung ihres Allgemeinbefindens, Zunahme des Appetites und eine erhebliche Gewichtszunahme, Angaben, die von uns durth den objektiven Befund bestätigt werden konnten.

Auch beim NSS. hat sich die schon beim Silbersalvarsan gemachte Erfahrung wiederholt, daß einzelne, durch Nummern gekennzeichnete Versuchsserien aus dem Speyerhaus verschieden vertragen wurden. Wenn auch der Unterschied bezüglich der Verträglichkeit der einzelnen Nummern nicht so in die Augen springend war, wie beim Silbersalvarsan, so haben sich doch oft Abweichungen auch 
beim NSS. ergeben. Wir haben festste...en können, daß ein und derselbe Patient das Präparat mit der Kontrolinummer a besser vertrug als das mit $b$ bezeichnete. Bei einer Serie war besonders der von den Patienten stets als ekelerregend geschilderte Salvarsangeschmack vorhanden.

Zubereitung der Lösung und Technik der Injektion. In den ersten 8 Monaten haben wir als Lösungsmittel stets $0,4 \%$ ige $\mathrm{NaCl}$-Lösung, die aus redestiliiertem und sterilisiertem Wasser mög. lichst täglich frisch im Laboratorium der Engel-Apotheke zu Leipzig hergestellt wurde, später erst auf Anregung von Geh.-Rat Kolle nur frisch redestilliertes und sterilisiertes Wasser benutzt, ohne übrigens den geringsten Unterschied bezüglich der Wirkung der Injektion feststellen zu köınen. Der Inhait der Ampullen wurde in $5 \mathrm{ccm}$ einer der obengenannten Lösungen aufgelöst. Das meist dunkelbraune Pulver - die Farben der einzelnen Serien variieren oft recht erheblich -, löst sich in wenig angewärmter Lösung sehr leicht und schnell, man muß jedoch sehr genau kontrollieren, ob die Lösung vollständig ist, da sich hin und wieder an den Glaswänden tungelöste Teile festsetzen, die, in die Blutbahn gebracht, zu sehr üblen Zufällen führen können. Die zur Injektion fertige NSS.-Lösung haben wir dann in eine $15 \mathrm{ccm}$ fassende Rekordspritze mit Glasstempel aufgesogen und in dieser die Mischung mit dem Novasurol vorgenommen.

Die Technik der Injektion wird als bekannt vorausgesetzt, sie bietet, da die Lösung tief dunkel ist, die für Ungeübte bekannten Schwierigkeiten. Neben die Vene in dás Gewebe gespritzte NSS.Lösung gibt sehr unangenehme Infiltrate. Ich empfehle dringend, sich bei der Injektion der einmal früher von Stern angegebenen Methode zu bedienen, die da vorschreibt, erst einige Kubikzentimeter Blut (wir nehmen $5 \mathrm{ccm}$ ) in die Spitze aufzusaugen, da mall erstens die Gewähr hat, wirklich in der Vene zu sein, und da vor allen Dingen die Mischung von Medikament und Blut schon in der Spritze vor sich geht, ein Umstand, der in puncto Schonung der Venenwand und nicht zu unmittelbarer Ueberführung der Lösung in die Blutbahn nicht hoch genug bewertet werden kann.

Die therapeutischen Erfolge. Die Einwirkung der NSS.Novasurolinjektionen auf ulzeröse Geschwüre, Plaques usw. ist prompt und sicher, wir sind gewöhnt, selbst größere ulzeröse Defekte nach wenigen Einspritzungen sich schnell reinigen und überhäuten zu sehen. Immer wieder zauberhaft ist die Wirkung bei den hochgradig entzündlichen Phimosen auf schankrös-syphilitischer Basis. Es ist erstaunlich, in welch kurzer Zeit sich diese oft monströs entzündlichen Veränderungen zur Norm zurückbilden. Bei diesen Fällen habe ich immer den stillen Wunsch, daB die Enragiertesten aus dem Lager der Salvarsangegner Zeugen der therapeutischen Erfolge und der Dankbarkeit der Patienten sein könnten.

Die abortiv mit 12 bzw. 14 kombinierten NSS.-Novasurolinjektionen behandelten Fälle sind, soweit sie sich nicht der Kontrolle entzogen haben, von uns weiter beobachtet und zunächst in Zwischenräumen von 4 zu 4 Wochen, später von 3 Monaten serologisch nachgeprüft worden, und zwar nach $W$ as s e rmann, zum großen Teil auch nach $S$ a ch s - G e orgi. Die Untersuchungen sind ausgeführt worden vom Prosektor des Krankenhauses St. Georg in Leipzig, Dr. Reinhardt, der unseren Arbeiten und unserem Material in dankenswerter Weise das größte Interesse entgegenbrachte. Sämtliche fortlaufend. : kontroliierten Fälle sind Wassermann- (Saclis-Georgi-) $\mathrm{n}$ e g a tiv geblieben, ein Befund, der doch mit an Sicherheit grenzender Wahrscheinlichkeit den Schluß zuläßt, daß wir von einer Heilung sprechen dürfen.

Etwas anders liegen die Dinge bei den bereits schwach positiven $(+=++)$ Kranken. Wenn auch von diesen der weitaus größte Teil bereits nach der ersten Kur Wassermann-negativ war, so ist doch ein gewisser, wenn auch kleiner Prozentsatz positiv geblieben. Wir lassen daher diese Art von Kranken gruildsätzlich 2 Kuren von je 14 kombinierten Injektionen mit einem Intervall von $\mathrm{h}$ ö chstens 3 Monaten machen und möglichst nach einem weiteren Vierteljähr noch eine Sicherheitskur von 10 Injektionen. Die Zeit von 13 Monaten ist naturgemäB zu kurz, um bezüglich der Heilungsfrage 'bei dieser Kategorie von Kranken irgendwelche bindenden Schlüsse zu ziehen, es wird der fortlaufenden Beobachtung dieser Patienten und der Weiterarbeit bedürfen, um feststellen zu können ob der von uns vorgeschlagene Heilplan genügt, um derartige Fälle dauernd zu heilen.

Die im vorgeschrittenen Sekundärstadium befindlichen Kranken (Wa.R. [Sa.-G.] +++-++++ ) mit Haut- oder Schleimhauterscheinungen müBten unbedingt drei Vollkuren vòn 14 kombinierten Injektionen in Intervallen von 2-3 Monaten und vielleicht 4 Monate nach der dritten Kur noch eine Sicherheitskur von 10 Spritzen durchführen. Auch bei Kranken im vorgeschrittenen S ekundärstadium konnten wir bei einem Drittel der Fälle bereits nach der ersten Kur einen völlıg negativen Wassermann notieren, ein Zeichen für die entschieden hochwertige Einwirkung des- NSS. in Verbindung mit Novasurol auf die Spirillen. Und es waren auffallenderweise oft die Fälle mit den schwersten Allgemeinerscheinungen, die bereits nach 14 Injektionen Wassermann-negativ wurden.

Bei den Erkrankungen der Spätsyphilisperiode (Tabes, Taboparalyse, Erkrankungen des Nerven- und GefäBsystems) sind die Erfolge sehr variabel. Recht schönen therapeutischen Resultaten, speziell im Anfangsstadium der Tabes und Taboparalyse (gastrische Krisen, lancierende Schmerzen, Gedächtnisschwäche, Neuritiden), stehen Fälle gegenüber, in denen keine oder nur schwache, nicht nennenswerte Erfolge zu verzeichnen waren. Ein älterer, früher aktiver Offizier mit ausgesprochenen taboparalytischen Erscheinungen war nach 2 Kuren im Jahre 1920 soweit hergestellt, daB er seine $B$ schäftigunE wieder aufnehmen konnte und sie heute noch ausübt. Ein anderèr Fall, ein Ausländer von 38 Jahren mit einem Gumma an der Schädelbasis am Ganglion Gasseri (Diagnnse: Geh.-Rat Pay r) ist nicht die Spur gebessert worden. Im allgemeinen haben wir den Eindruck, daB die noch Wassermann-positiven Fälle der Spätsyphilis eher zu beeinflussen sind als die Wassermann-negativen. Jedoch ist auch hier die Beobachtungszeit zu kurz, es soll später eingehender darüber berichtet werden.

Zusammenfassung. Wir scheinen dem. Ziele, ein leicht lösliches Salvarsanpräparat zu schaffen, das bei geringster Toxizität und guter Verträglichkeit die sichersten und höchstwertigen spirillentötenden Eigenschaften hat, im Neosilbersalvarsan erheblich näherge rückt zu sein. Wir habeiı bei einem ausgesuchten Material von 200 Fällen mit etwa 3000 Injektionen von NSS. mit Novasurol in einzeitiger Kombination Erfolge bei der Behandlung' ailer Stadien der Syphilis gesehen, wie bei keinem der früheren Präparate "und bei keiner anderen Kombination von Salvarsan und Quecksilber. Die recht beacht liche Erfahrung, daß diese günstigen therapeutischen Erfolge erzielt werden konnten olnne nennenswerte Nachteile und Störungen fiir den Organisinus, eröffnet eine ermutigende Perspektive tür das Speyerhaus, auf dem beschrittenen Wege weiterzugehen und weiterzuforschen. 